PROCEEDINGS OF THE

AMERICAN MATHEMATICAL SOCIETY

Volume 128, Number 5, Pages 1527-1530

S 0002-9939(99)05372-1

Article electronically published on August 17, 1999

\title{
PARALLELIZABILITY OF COMPLEX PROJECTIVE STIEFEL MANIFOLDS
}

\author{
L. ASTEY, S. GITLER, E. MICHA, AND G. PASTOR \\ (Communicated by Ralph Cohen)
}

\begin{abstract}
The question of parallelizability of the complex projective Stiefel manifolds is settled.
\end{abstract}

\section{INTRODUCTION}

The motivation for this note derives from our work [2], where a description of the cohomology of the complex projective Stiefel manifolds $P W_{n, k}$ is given, and from our interest in the question of parallelizability of certain families of manifolds 3].

The manifold $P W_{n, k}$ is the quotient space of the free circle action on the complex Stiefel manifold $W_{n, k}$ of orthonormal $k$-frames in complex $n$-space given by $z\left(v_{1}, \ldots, v_{k}\right)=\left(z v_{1}, \ldots, z v_{k}\right)$. Our result is as follows.

Theorem. If $k<n-1$, then $P W_{n, k}$ is not stably parallelizable; $P W_{n, n-1}$ is parallelizable, except $P W_{2,1}=S^{2}$; and $P W_{n, n}$ is the projective unitary group, and so is parallelizable.

\section{Proof}

Consider the standard inclusion of $U(k) \times U(n-k)$ in $U(n)$ and regard $S^{1} \times$ $U(n-k)$ as the subgroup

$$
\left\{\left(\begin{array}{cc}
z I & 0 \\
0 & A
\end{array}\right) \mid z \in S^{1} \text { and } A \in U(n-k)\right\}
$$

of $U(k) \times U(n-k)$. Then the map $U(n) \longrightarrow W_{n, k}$ that takes the first $k$ columns induces a diffeomorphism

$$
P W_{n, k} \cong U(n) / S^{1} \times U(n-k)
$$

and we obtain a principal bundle

$$
P U(k) \longrightarrow P W_{n, k} \stackrel{q}{\longrightarrow} G r_{k}\left(\mathbb{C}^{n}\right)
$$

where $G r_{k}\left(\mathbb{C}^{n}\right)$ is the Grassmann manifold of complex $k$-planes in $\mathbb{C}^{n}$ and $q$ sends a given point of $P W_{n, k}$ to the $k$-plane generated by any $k$-frame representing the given point. Let $\xi$ and $\xi^{\perp}$ be the canonical $k$-plane and $(n-k)$-plane bundles on

Received by the editors June 19, 1998.

1991 Mathematics Subject Classification. Primary 57R25.

The second author was partially supported by an NSF grant. 
$G r_{k}\left(\mathbb{C}^{n}\right)$, so that $\xi \oplus \xi^{\perp} \cong n$, the trivial complex bundle of dimension $n$. There is an isomorphism of complex bundles

$$
T G r_{k}\left(\mathbb{C}^{n}\right) \cong \operatorname{Hom}_{\mathbb{C}}\left(\xi, \xi^{\perp}\right),
$$

where $T$ denotes the tangent bundle. This is easy to prove as in [5, p. 169]. If $P$ is a $k$-plane in $\mathbb{C}^{n}$ define a map

$$
\operatorname{Hom}_{\mathbb{C}}\left(P, P^{\perp}\right) \longrightarrow G r_{k}\left(\mathbb{C}^{n}\right)
$$

by $\varphi \mapsto$ graph of $\varphi$. The differential at the origin is an isomorphism

$$
\operatorname{Hom}_{\mathbb{C}}\left(P, P^{\perp}\right)=T_{0} \operatorname{Hom}_{\mathbb{C}}\left(P, P^{\perp}\right) \stackrel{\cong}{\longrightarrow} T_{P} G r_{k}\left(\mathbb{C}^{n}\right)
$$

and this determines a bundle isomorphism (2.2). Since (2.1) is a principal bundle, the bundle of tangents along the fibre is trivial, and we have an isomorphism of real vector bundles

$$
T P W_{n, k} \cong q^{*} T G r_{k}\left(\mathbb{C}^{n}\right) \oplus\left(k^{2}-1\right) .
$$

Here $k^{2}-1$ denotes a real trivial vector bundle of dimension $k^{2}-1$.

Let $L$ be the complex line bundle on $P W_{n, k}$ associated with the bundle $\pi: W_{n, k} \rightarrow P W_{n, k}$; let $L_{i}$ be the complex line bundle whose fibre over a point of $P W_{n, k}$ is the subspace generated by the $i$ th vector on any frame representing the point.

The total space of $L$ is the quotient $W_{n, k} \times_{S^{1}} \mathbb{C}$ of $W_{n, k} \times \mathbb{C}$ by the equivalence relation that identifies $\left(v_{1}, \ldots, v_{k}, w\right)$ with $\left(z v_{1}, \ldots, z v_{k}, z^{-1} w\right)$. The map

$$
\varphi_{i}: W_{n, k} \times_{S^{1}} \mathbb{C} \longrightarrow P W_{n, k} \times \mathbb{C}^{n}
$$

given by $\varphi_{i}\left(v_{1}, \ldots, v_{k}, w\right)$ ((line generated by $\left.\left.v_{i}\right), w v_{i}\right)$ is a vector bundle monomorphism with $\operatorname{im} \varphi_{i}=L_{i}$. Thus $L_{i} \cong L$ for all $1 \leq i \leq k$, and so

$$
q^{*} \xi \cong k L,
$$

the Whitney sum of $k$ copies of $L$, since it is clear that $q^{*} \xi=L_{1} \oplus \cdots \oplus L_{k}$. Then, if $\xi^{*}=\operatorname{Hom}_{\mathbb{C}}(\xi, \mathbb{C})$,

$$
q^{*} T G r_{k}\left(\mathbb{C}^{n}\right) \cong q^{*} \operatorname{Hom}_{\mathbb{C}}\left(\xi, \xi^{\perp}\right) \cong q^{*}\left(\xi^{*} \otimes_{\mathbb{C}} \xi^{\perp}\right) \cong k L^{*} \otimes_{\mathbb{C}} q^{*} \xi^{\perp} .
$$

Since $\xi \oplus \xi^{\perp} \cong n$ we have from (2.4)

$$
k L \oplus q^{*} \xi^{\perp} \cong n
$$

and so, in $K U\left(P W_{n, k}\right)$,

$$
\begin{aligned}
q^{*} T G r_{k}\left(\mathbb{C}^{n}\right) & =k L^{*}(n-k L) \\
& =n k L^{*}-k^{2} .
\end{aligned}
$$

Thus, we see from (2.3) that $T P W_{n, k}$ is stably equivalent to $n k r\left(L^{*}\right)$, where $r\left(L^{*}\right)$ denotes the "realification" of $L^{*}$. Then the Pontrjagin class

$$
p\left(P W_{n, k}\right)=p\left(n k r\left(L^{*}\right)\right)=\left(1+x_{0}^{2}\right)^{n k}
$$

where $x_{0}=-c_{1}\left(L^{*}\right)$.

Assume $k<n-1$. Then $W_{n, k}$ is 4 -connected, so the Gysin sequence of the sphere bundle $\pi: W_{n, k} \longrightarrow P W_{n, k}$ shows that $H^{4}\left(P W_{n, k} ; \mathbb{Z}\right) \cong \mathbb{Z}$ is generated by $x_{0}^{2}$. From (2.7),

$$
p_{1}\left(P W_{n, k}\right)=n k x_{0}^{2} \neq 0,
$$

so $P W_{n, k}$ is not stably parallelizable. 
Consider the case $k=n-1$. Then (2.5) becomes

$$
(n-1) L \oplus q^{*} \xi^{\perp} \cong n
$$

and this implies

$$
n L^{*} \cong(n-1) \oplus E
$$

where $E=L^{*} \otimes_{\mathbb{C}} q^{*} \xi^{\perp}$. Applying the second exterior power operator $\lambda^{2}$ to (2.8) we obtain

$$
\begin{aligned}
\left(\begin{array}{c}
n \\
2
\end{array}\right)\left(L^{*}\right)^{2} \cong \lambda^{2}\left(n L^{*}\right) & \cong \lambda^{2}((n-1) \oplus E) \\
& \cong\left(\begin{array}{c}
n-1 \\
2
\end{array}\right) \oplus(n-1) E .
\end{aligned}
$$

Let $u=L^{*}-1 \in K U\left(P W_{n, n-1}\right)$. Then a straightforward calculation using (2.8) and (2.9) shows that

$$
\left(\begin{array}{c}
n \\
2
\end{array}\right) u^{2}=0
$$

Let

$$
c: K O\left(P W_{n, n-1}\right) \longrightarrow K U\left(P W_{n, n-1}\right)
$$

be the "complexification" homomorphism; it satisfies $\operatorname{cr}(F)=F+F^{*}$ for any complex vector bundle $F$.

Then

$$
\begin{aligned}
\operatorname{cr}(u) & =L^{*}+L-2 \\
& =1+u+\frac{1}{1+u}-2 \\
& =\frac{u^{2}}{1+u},
\end{aligned}
$$

so that $c\left(\left(\begin{array}{l}n \\ 2\end{array}\right) r(u)\right)=0$. Since ker $c$ is well known to consist of elements of order 2 we obtain

$$
n(n-1) r(u)=0
$$

in $K O\left(P W_{n, n-1}\right)$. By (2.3) and (2.6) we now have

$$
T P W_{n, n-1}-\operatorname{dim} P W_{n, n-1}=n(n-1) r(u)=0,
$$

so $P W_{n, n-1}$ is stably parallelizable.

To show $P W_{n, n-1}$ is parallelizable for $n>2$ we appeal to a theorem proved in [6] and [4] that states that if an $(m-1)$-dimensional manifold is stably parallelizable, then either it is parallelizable or it admits exactly the same number of linearly independent tangent vector fields as the $(m-1)$-sphere. This immediately implies the parallelizability of $P W_{3,2}$, since it has dimension seven and $S^{7}$ is parallelizable. By [1] the $(m-1)$-sphere admits exactly $\rho(m)-1$ linearly independent tangent vector fields, where $\rho$ is the numerical function given by $\rho(m)=8 a+2^{b}$ if $m=2^{4 a+b}$. (odd integer) with $0 \leq b \leq 3$. The parallelizability of $P W_{n, n-1}$ for $n>3$ is a consequence of (2.3) and the following lemma.

Lemma 2.10. If $n>3$, then $\rho\left(n^{2}-1\right)<(n-1)^{2}$.

Proof. Note first that if $n$ is even, then $\rho\left(n^{2}-1\right)=1$, so the lemma holds in this case. If $n=2 m+1$, then $m>1$ and we must prove that

$$
\rho(4 m(m+1))<4 m^{2} .
$$


Let $\nu$ be the numerical function defined by $t=2^{\nu(t)}$.(odd integer). It is easy to verify that $\rho(t) \leq 2 \nu(t)+2$ and $\nu(t) \leq t-1$ for all $t>0$. Then

$$
\begin{aligned}
\rho(4 m(m+1)) & \leq 2 \nu(4 m(m+1))+2 \\
& =2(2+\nu(m)+\nu(m+1))+2 \\
& \leq 4 m+4 \\
& <4 m^{2},
\end{aligned}
$$

where the last inequality holds because $m>1$.

\section{REFERENCES}

[1] J.F. Adams, Vector fields on spheres, Ann. of Math. 75 (1962), 603-632. MR 25:2614

[2] L. Astey, S. Gitler, E. Micha, G. Pastor, Cohomology of complex projective Stiefel manifolds, to appear in Canad. J. Math.

[3] L. Astey, E. Micha, G. Pastor, Homeomorphism and diffeomorphism types of Eschenburg spaces, Diff. Geom. Appl. 7 (1997), 41-50. MR 98h:53072

[4] G.E. Bredon and A. Kosinski, Vector fields on $\pi$-manifolds, Ann. of Math. (2) 84 (1966), 85-90. MR 34:823

[5] J. Milnor and J.D. Stasheff, Characteristic classes, Annals of Mathematics Studies No. 76. Princeton Univ. Press, Princeton, New Jersey. MR 55:13428

[6] E. Thomas, Cross-sections of stably equivalent vector bundles, Quart. J. Math. Oxford Ser. (2) 17 (1966) 53-57. MR 33:4933

(L. Astey and E. Micha) Centro de Investigación del IPN, Apartado Postal 14-740, MÉXICO 07000 D.F.

E-mail address: lastey@math.cinvestav.mx

E-mail address: emicha@math.cinvestav.mx

(S. Gitler) Department of Mathematics, University of Rochester, Rochester, New YORK 14627-0001

E-mail address: sgitler@math.rochester.edu

(G. Pastor) Instituto Tecnológico Autónomo de México, Rio Hondo No. 1, San Angel, MÉXICO 01000 D.F.

E-mail address: pastor@gauss.rhon.itam.mx 